\title{
EXPLORANDO LOS BENEFICIOS DE LOS RÍOS SECOS PARA EL BIENESTAR HUMANO: UNA PERSPECTIVA SOCIAL
}

\begin{abstract}
REsumen
Los ríos secos son cauces cuyo hábitat es terrestre, están desconectados de las aguas subterráneas, únicamente transportan agua que evacuan rápidamente tras fuertes lluvias esporádicas y, por lo tanto, no albergan organismos acuáticos. La ausencia permanente de agua en estos cauces constituye la principal razón por la que gestores y sociedad civil, en general, los consideran inútiles e improductivos, y son uno de los ecosistemas más impactados del mundo. Este trabajo afronta tres objetivos principales. En primer lugar, revisar la diversidad de servicios ecosistémicos que los ríos secos proporcionan al bienestar humano utilizando el marco conceptual propuesto por la Plataforma Intergubernamental CienciaPolítica sobre Biodiversidad y Servicios Ecosistémicos (IPBES). En segundo lugar, detectar los impulsores directos e indirectos de cambio, claves que afectan a la capacidad de estos ríos para generar un flujo sostenible de servicios. En tercer lugar, aportar información sobre la percepción social que la ciudadanía de la región de Murcia (España) tienen sobre los ríos secos aplicando la técnica de libre listado (freelisting). El trabajo se basa en la revisión bibliográfica de la literatura científica (sin menospreciar la literatura gris) que aporta evidencias sobre los beneficios de los ríos secos a la sociedad. Los resultados muestran que estos ecosistemas contribuyen al bienestar humano, no tanto por su alta capacidad de
\end{abstract}

\footnotetext{
a Catedrática de Ecología. Departamento de Ecología e Hidrología, Universidad de Murcia. Campus de Espinardo. 30100 Murcia. charyvag@um.es. https://orcid.org/0000-0002-2225-0727.

b Contrato predoctoral de la Fundación Séneca (Ref: 20754/FPI/18). Departamento de Ecología e Hidrología, Universidad de Murcia. Campus de Espinardo. 30100 Murcia. nestor.nicolas@um.es

c Catedrática de Ecología. Departamento de Ecología e Hidrología, Universidad de Murcia. Campus de Espinardo. 30100 Murcia. mlsuarez@um.es. https://orcid.org/0000-0003-0420-4919.
}

Fecha de recepción: 15-04-2021. Fecha de aceptación: 12-11-2021. 
proveer servicios ecosistémicos sino por la fuerte relación y colaboración que, a lo largo de la historia, ha existido entre las comunidades que viven en torno a estos ecosistemas y los recursos que proveen. Impulsores de cambio de naturaleza social y económica están alterando los modelos de sostenibilidad que mantenían un alto grado de resiliencia de estos socio-ecosistemas. La consulta a la ciudadanía reveló paradójicamente que, aunque el conocimiento actual sobre los ríos secos no es completo y su utilidad está infravalorada, existe un valor sociocultural fuertemente arraigado.

Palabras clave: contribuciones de la naturaleza a la gente; freelisting; impulsores de cambio; Murcia (España); servicios ecosistémicos; sistema socioecológico.

\title{
EXPLORING THE BENEFITS OF DRY RIVERS FOR HUMAN WELL-BEING: A SOCIAL PERSPECTIVE
}

\begin{abstract}
Dry rivers are channels whose usual habitat is terrestrial. They are disconnected from groundwater, only transport water that is quickly evacuated after sporadic heavy rains and, therefore, do not harbor aquatic organisms. The permanent absence of water in these channels is the main reason why both managers and society consider them useless and unproductive, being one of the most impacted ecosystems in the world. This work focuses on three main objectives. Firstly, we review the diversity of ecosystem services that these ecosystems provide to human well-being using the conceptual framework proposed by the Intergovernmental Science-Policy Platform on Biodiversity and Ecosystem Services (IPBES). Secondly, we identify the key direct and indirect drivers of change affecting the ability of dry rivers to provide a sustainable flow of services. Thirdly, we provide information on the social perception that citizens of the Region of Murcia (Spain) have about dry rivers by applying the freelisting method. This work is based on the bibliographic review of the scientific literature (without underestimating gray literature) that provides evidence about the benefits of dry rivers to people. The results show that these ecosystems contribute to human well-being not only because of their high capacity to provide ecosystem services, but also because of the strong relationship and collaboration that, throughout history, has existed between the human communities who inhabit dry rivers and the natural resources provided by them. Social and economic drivers of change are altering the sustainability models that maintained a high degree of resilience of these socio-ecosystems. The social analysis revealed a limited knowledge about dry rivers and their benefits but also, a high sociocultural value for their inhabitants.
\end{abstract}

KeYwords: nature's contributions to people; freelisting; drivers of change; Murcia (Spain); ecosystem services; socio-ecological system. 


\section{INTRODUCCIÓN}

Los ríos secos son cauces que permanecen secos durante todo el ciclo hidrológico anual excepto cuando fuertes lluvias esporádicas generan una crecida que evacua el agua rápidamente. Están desconectados de las aguas subterráneas y, por lo tanto, no albergan vida acuática (Vidal-Abarca et al., 2020). Los ríos secos constituyen el extremo hidrológico del gradiente que se puede establecer dentro del complejo mundo de los ríos no perennes: desde los ríos intermitentes (aquellos que cesan su flujo de agua durante alguna fase del ciclo hidrológico anual), pasando por los ríos efímeros (aquellos que cesan su flujo de agua de forma impredecible a lo largo del ciclo hidrológico anual) (Datry et al., 2017a). Resulta obvio que el hábitat natural de los ríos secos es terrestre, por lo que tanto su biodiversidad como los procesos ecológicos que los caracterizan presentan mayor similitud con lo que ocurre en el ámbito terrestre, que en el acuático. Paradójicamente, los ríos secos se generan y dinamizan por la fuerza del agua que fluye tras las avenidas (García et al., 1999; Gordon et al., 2004), de manera que, en un contexto territorial, forman parte de la red fluvial de las cuencas de drenaje.

Aunque los ríos secos son especialmente abundantes en las regiones más áridas del planeta (Mabbutt, 1977; Bull \& Kirkby, 2002; Levick et al., 2008), no son exclusivos de ellas (Larned et al., 2010; Schneider et al., 2017). Los ríos secos se encuentran en todos los ámbitos climáticos (Stanley et al., 1997; Datry et al., 2014), incluidas las regiones húmedas y subhúmedas (Fritz et al., 2006; Buttle et al., 2012). Sin embargo, no existen datos precisos sobre su cuantía y distribución a escala global. Actualmente, los datos más ajustados son los proporcionados por Raymond et al. (2013), quienes estiman que el $69 \%$ de los cauces de primer orden por debajo de los $60^{\circ}$ de latitud y aproximadamente el $34 \%$ de los ríos de mayor orden fluyen de forma intermitente, donde evidentemente están incluidos los ríos secos.

La ausencia de un flujo permanente de agua en los ríos secos los ha vuelto invisibles a ojos de gestores del agua, del territorio y de la sociedad, que los consideran inútiles e improductivos (DeLucio \& Múgica, 1994; García-Llorente et al., 2012). Únicamente se les presta atención cuando fuertes lluvias los desbordan y provocan daños humanos y materiales de consideración (Di Baldassarre et al., 2010; Machado et al., 2017). Esto ha derivado en una visión peyorativa y nada beneficiosa de los ríos secos, por lo que probablemente sean uno de los ecosistemas fluviales más impactados del planeta (Jacobson et al., 1995; Seely et al., 2003; Gómez et al., 2005; Levick et al., 2008).

En este contexto se enmarcan los objetivos del presente trabajo. En primer lugar, se pretende revisar la capacidad de los ríos secos para proveer servicios ecosistémicos a la sociedad a través de la información aportada por publicaciones científicas. A excepción de dos trabajos previos realizados por Datry et al. (2017b) y Koundouri et al. (2017) para los ríos intermitentes y algunos datos aportados por Vidal-Abarca et al. (2020) para los ríos secos, no existe un análisis global sobre los beneficios potenciales que estos ecosistemas pueden suministrar a la sociedad. Probablemente el desconocimiento de la población humana sobre los beneficios de los ríos secos para el bienestar humano sea una de las razones para ignorarlos o considerarlos sistemas baldíos y degradados. En segundo lugar, detectar, mediante la revisión bibliográfica y de forma genérica, los impulsores directos e indirectos de cambio claves 
que están afectando a la capacidad de estos ecosistemas para producir un flujo sostenible de servicios. Finalmente, a una escala local, se pretende aportar información sobre la percepción social y el conocimiento que tiene la ciudadanía de la Región de Murcia sobre los ríos secos.

\section{Metodología}

Entre los diferentes marcos conceptuales existentes para analizar los componentes básicos y las interacciones entre la naturaleza y el sistema social (MA, 2005; TEEB, 2010; EME, 2011; Haines-Young $\&$ Potschin, 2013), en este caso se ha aplicado el propuesto por la Plataforma Intergubernamental Ciencia-Política sobre Biodiversidad y Servicios Ecosistémicos (IPBES) (Díaz et al., 2015). Este marco conceptual incorpora la noción de "contribuciones de la naturaleza a la gente" (con sus siglas en inglés NCP) (Díaz et al., 2018), y las clasifica en tres amplios grupos: contribuciones materiales, no materiales y de regulación para una buena calidad de vida. El concepto NCP es equivalente al de servicios ecosistémicos, pero incorpora un conjunto más amplio y diverso de sistemas de conocimiento (por ejemplo, ciencias sociales y humanidades), distintas visiones del mundo (por ejemplo, el conocimiento local e indígena) y más partes interesadas para fortalecer las relaciones ciencia-política sobre las personas y la naturaleza, con lo que aumenta la inclusión y la pluralidad (Kadykalo et al., 2019). Además, en este marco se reconoce que los NCP pueden ser tanto positivos como negativos, aunque en este trabajo se hace especial énfasis en los positivos. Así pues, este esquema conceptual es más útil para cumplir los objetivos del presente trabajo, dado que los ríos secos se ubican mayoritariamente en ámbitos áridos y semiáridos, donde la población humana ha generado a lo largo de la historia un conocimiento ecológico local propio para utilizar de forma singular y sostenible los recursos que los ríos les proporcionan.

Para cumplir estos objetivos se realizó una búsqueda exhaustiva de la bibliografía científica en distintas bases de datos (ISI web del conocimiento; Google Scholar; Scopus). No obstante, conscientes de que la literatura gris aporta mucha información no distribuida por los canales habituales y que puede contribuir a un mayor conocimiento tanto de los NCP que proveen los ríos secos (Mengist et al., 2020) como de las experiencias que la gente obtiene de estos, se incorporó a esta revisión cuando se consideró relevante.

Los términos de la búsqueda siempre incluían dry river y/o distintos vocablos con los que los ríos secos son habitualmente nombrados en la literatura (por ejemplo, dryland, ephemeral, episodic o seasonal; seguido de river, o stream, o system, o channel). Adicionalmente, la búsqueda también incluyó vocablos de uso geográficamente más restringido, como wadis, creek, ramblas, balka, etc. El objeto de la búsqueda fue encontrar evidencias escritas de todos los NCP que los ríos secos fueran capaces de suministrar, así como de los impulsores de cambio que los afectaban. Esta forma de rastreo de las evidencias llevaba consigo la lectura detenida del texto completo con el fin de confirmar que el trabajo se refería a ríos secos, tal como se han definido anteriormente. Además, las evidencias sobre los NCP y los impulsores de cambio fueron agrupadas en las categorías descritas por el marco IPBES. 
Para cumplir el tercer objetivo, es decir, aportar información sobre la percepción y el conocimiento que la ciudadanía asigna a los ríos secos, se utilizó la técnica de libre listado (freelisting) (Bieling et al., 2014; Kelemen et al., 2016). Se trata de un método de consulta directa que tiene por objeto obtener respuestas espontáneas y no dirigidas. La cuestión que se propuso fue: "Describe las ramblas con tres o cuatro palabras" y fue dirigida a 177 ciudadanos de la Región de Murcia que utilizan el término de rambla para identificar a los ríos secos. Las respuestas obtenidas fueron cuantificadas contabilizando el número de veces que aparecía repetida cada palabra. Además, se identificaron varios criterios de respuesta; es decir, se analizó la naturaleza de cada palabra empleada por la ciudadanía. De esta manera, se codificaron y agruparon las respuestas en grupos temáticos que permitiesen sintetizar la información y facilitar la interpretación de los datos.

\section{ResUltados Y Discusión}

\section{Revisando los beneficios de los ríos secos para una buena calidad de vida}

En la revisión bibliográfica realizada se encontraron ejemplos y/o evidencias para 17 de las 18 categorías incluidas en los tres grupos de NCP usadas en el marco conceptual de IPBES (tabla 1) (por razones obvias, no se encontraron referencias para el servicio de regulación de la acidificación de los océanos). En muchos casos, las NCP están fuertemente soportadas por las evidencias encontradas. Las contribuciones no materiales incluyen todas las experiencias subjetivas, físicas y psicológicas, tanto individuales como colectivas, que la gente experimenta en contacto con la naturaleza. En este sentido, la literatura aporta muchos ejemplos sobre las oportunidades que los ríos secos están ofreciendo para la observación y el aprendizaje del medio natural (Alberdi, 2011; Safriel \& Adeel 2015), para aumentar el conocimiento sobre especies presentes y procesos que ocurren en estos ecosistemas (Sánchez-Montoya et al., 2016; Arce et al., 2019) o como espacio de inspiración para escritores, poetas, pintores y otros artistas (Neruda, 1976; Región de Murcia Digital; Andreu-Lara \& Ojeda-Rivera, 2019). Además, los ríos secos son espacios especialmente utilizados para desarrollar muchas actividades de recreo y ocio en contacto con la naturaleza (Gómez et al., 2005; Steward et al. 2012; Stubbington et al., 2020) que son beneficiosas para la salud física y mental de las personas (Samakov, 2017), o simplemente para el disfrute de paisajes (Seely et al., 2003; Andreu-Lara \& Ojeda-Rivera, 2019), algo que facilita la relajación y curación (Teff-Seker \& Orenstein, 2019). Los ríos secos son espacios de conexión social, comunican pueblos cercanos (Gómez et al., 2005) y generan en las personas sentimientos de pertenencia, que ligan a tradiciones, leyendas, rituales o experiencias espirituales y religiosas (Safriel \& Adeel, 2005). 
Tabla 1. Evidencias y/o ejemplos de los beneficios de los ríos secos para una buena calidad de vida basado en el marco conceptual IPBES.

Fuente: Díaz et al., 2018.

\begin{tabular}{|c|c|c|}
\hline $\begin{array}{l}\text { Contribuciones de los } \\
\text { ríos secos al bienestar } \\
\text { humano (NCP) }\end{array}$ & Ejemplos/evidencias & Referencias bibliográficas \\
\hline \multicolumn{3}{|c|}{ Contribuciones de regulación } \\
\hline \multirow{3}{*}{$\begin{array}{l}\text { Creación y } \\
\text { mantenimiento } \\
\text { de hábitats }\end{array}$} & $\begin{array}{l}\text { La vegetación de los ríos secos potencia los procesos } \\
\text { de sedimentación generando nuevos hábitats }\end{array}$ & $\begin{array}{l}\text { Sandercock et al. (2007); } \\
\text { Hinchman (2013) }\end{array}$ \\
\hline & $\begin{array}{l}\text { La vegetación de los ríos secos ralentiza la } \\
\text { transferencia de sedimentos río abajo manteniendo } \\
\text { hábitats terrestres }\end{array}$ & Sandercock et al. (2007) \\
\hline & $\begin{array}{l}\text { Los ríos secos son hábitats para las comunidades } \\
\text { de muchos invertebrados terrestres }\end{array}$ & $\begin{array}{l}\text { Sánchez-Montoya et al. } \\
\text { (2016) }\end{array}$ \\
\hline \multirow{2}{*}{$\begin{array}{l}\text { Polinización y } \\
\text { dispersión de semillas } \\
\text { y otros propágulos }\end{array}$} & $\begin{array}{l}\text { Dispersión de semillas por animales (por ejemplo } \\
\text { serpientes de cascabel) (zoocoria) }\end{array}$ & Reiserer et al. (2018) \\
\hline & Dispersión de semillas por el viento (anemocoria) & Thompson et al. (2014) \\
\hline \multirow{2}{*}{$\begin{array}{l}\text { Regulación de la } \\
\text { calidad del aire }\end{array}$} & $\begin{array}{l}\text { El tiempo de residencia del carbono orgánico } \\
\text { en los lechos de los ríos secos es mucho mayor } \\
\text { que en los bosques }\end{array}$ & Farage et al. (2003) \\
\hline & $\begin{array}{l}\text { Secuestro de nitrógeno total desde la atmósfera } \\
\text { en los lechos de los ríos secos }\end{array}$ & Scholz et al. (2002) \\
\hline $\begin{array}{l}\text { Regulación } \\
\text { del microclima }\end{array}$ & $\begin{array}{l}\text { La vegetación de los ríos secos genera un microclima } \\
\text { más suave porque disminuyen la temperatura local }\end{array}$ & $\begin{array}{l}\text { Valenzuela et al. (2011); } \\
\text { Roman (2016) }\end{array}$ \\
\hline \multirow{2}{*}{$\begin{array}{l}\text { Regulación de la } \\
\text { cantidad, localización y } \\
\text { tiempo de permanencia } \\
\text { del agua dulce }\end{array}$} & $\begin{array}{l}\text { Los lechos de algunos ríos secos son zonas } \\
\text { de infiltración del agua }\end{array}$ & Suftin et al. (2014) \\
\hline & $\begin{array}{l}\text { Especies vegetales indicadoras de la presencia } \\
\text { de agua subterránea con el mezquite (Prosopis sp.) } \\
\text { o los azufaitos (Ziziphus lotus) }\end{array}$ & $\begin{array}{l}\text { CONAZA-INE (1994); } \\
\text { López-Rodríguez et al. } \\
(2020)\end{array}$ \\
\hline \multirow{2}{*}{$\begin{array}{l}\text { Regulación de la } \\
\text { calidad del agua }\end{array}$} & $\begin{array}{l}\text { En los sedimentos de los ríos secos se pierde el fósforo } \\
\text { total }\end{array}$ & Scholz et al. (2002) \\
\hline & $\begin{array}{l}\text { La desnitrificación, vía microbiana, es la pérdida de } \\
\text { nitrógeno más importante en los ríos secos }\end{array}$ & Whitford (2002) \\
\hline
\end{tabular}




\begin{tabular}{|c|c|c|}
\hline $\begin{array}{l}\text { Contribuciones de los } \\
\text { ríos secos al bienestar } \\
\text { humano (NCP) }\end{array}$ & Ejemplos/evidencias & Referencias bibliográficas \\
\hline \multirow{5}{*}{$\begin{array}{l}\text { Formación, protección } \\
\text { y descontaminación } \\
\text { del suelos y sedimentos }\end{array}$} & $\begin{array}{l}\text { La vegetación que crece en los ríos secos deposita } \\
\text { materia orgánica que facilita la formación de suelo }\end{array}$ & Ríos Saucedo et al. (2013) \\
\hline & $\begin{array}{l}\text { La vegetación de los ríos secos aumenta su resistencia } \\
\text { a la erosión }\end{array}$ & $\begin{array}{l}\text { Sandercock et al. (2007); } \\
\text { Hinchman (2013) }\end{array}$ \\
\hline & $\begin{array}{l}\text { Los microorganismos de origen terrestre aceleran } \\
\text { la descomposición de la materia orgánica } \\
\text { en los ríos secos }\end{array}$ & Arce et al. (2019) \\
\hline & $\begin{array}{l}\text { La fotodegradación es una de las principales vías } \\
\text { de degradación de la materia orgánica en ríos secos }\end{array}$ & $\begin{array}{l}\text { Almagro et al. (2017); Del } \\
\text { Campo \& Gómez (2016) }\end{array}$ \\
\hline & $\begin{array}{l}\text { Secuestro de carbono inorgánico en los lechos } \\
\text { de los ríos secos vía formación de carbonatos }\end{array}$ & Farage et al. (2003) \\
\hline \multirow{2}{*}{$\begin{array}{l}\text { Regulación de } \\
\text { perturbaciones y } \\
\text { eventos extremos }\end{array}$} & $\begin{array}{l}\text { Los tramos trenzados de los ríos secos pueden ser más } \\
\text { resilientes a las perturbaciones naturales }\end{array}$ & Suftin et al. (2014) \\
\hline & $\begin{array}{l}\text { Los ríos secos contribuyen a minimizar los efectos } \\
\text { devastadores de las avenidas }\end{array}$ & Machado et al. (2017) \\
\hline \multirow{2}{*}{$\begin{array}{l}\text { Regulación de } \\
\text { organismos perjudiciales } \\
\text { y procesos biológicos }\end{array}$} & $\begin{array}{l}\text { Algunas plantas invasoras en los ríos secos pueden ser } \\
\text { controladas por pequeños roedores }\end{array}$ & Williams et al. (2013) \\
\hline & Biorremediación por comunidades microbianas & Burns et al. (2013) \\
\hline \multicolumn{3}{|l|}{ Contribuciones materiales } \\
\hline Energía & $\begin{array}{l}\text { El procesado de muchas plantas de los ríos secos (por } \\
\text { ejemplo, el mezquite: Prosopis sp.) produce carbón }\end{array}$ & Ríos Saucedo et al. (2013) \\
\hline \multirow{5}{*}{ Alimentos y piensos } & $\begin{array}{l}\text { En muchos ríos secos se cultivan cereales para } \\
\text { alimento humano o del ganado }\end{array}$ & Rodríguez Vaquero (2007) \\
\hline & $\begin{array}{l}\text { La vegetación de los ríos secos sirve de alimento al } \\
\text { ganado ovino y caprino }\end{array}$ & $\begin{array}{l}\text { Little et al. (1990); Arenas } \\
\text { (2012) }\end{array}$ \\
\hline & $\begin{array}{l}\text { Diversas especies vegetales presentes en los ríos secos } \\
\text { son utilizadas como alimento del ganado, por ejemplo, } \\
\text { el palo de fierro (Olneya tesota) en México }\end{array}$ & Parra et al. (2013) \\
\hline & $\begin{array}{l}\text { Diversas especies vegetales presentes en los ríos } \\
\text { secos son utilizadas para alimentación humana } \\
\text { (por ejemplo, recolección de espárragos trigueros: } \\
\text { Asparagus acutifolius) }\end{array}$ & Salinas \& Guirado (2002) \\
\hline & Caza de conejos para alimentación humana & Sánchez et al. (2004) \\
\hline
\end{tabular}




\begin{tabular}{|c|c|c|}
\hline $\begin{array}{l}\text { Contribuciones de los } \\
\text { ríos secos al bienestar } \\
\text { humano (NCP) }\end{array}$ & Ejemplos/evidencias & Referencias bibliográficas \\
\hline \multirow{3}{*}{$\begin{array}{l}\text { Materiales, animales } \\
\text { de compañía y labor }\end{array}$} & Extracción de arenas y gravas & Madyise (2013) \\
\hline & Extracción de sal en ríos secos & $\begin{array}{l}\text { Albaladejo-García \& } \\
\text { Gómez-Espín (2016) }\end{array}$ \\
\hline & Extracción de oro en ríos secos & Crane (2014) \\
\hline $\begin{array}{l}\text { Recursos medicinales, } \\
\text { bioquímicos y genéticos }\end{array}$ & $\begin{array}{l}\text { Muchas plantas que crecen en los ríos secos tienen } \\
\text { usos medicinales }\end{array}$ & $\begin{array}{l}\text { Ahmad et al. (2004); } \\
\text { Arenas (2012); Martínez- } \\
\text { Yoshino et al. (2021) }\end{array}$ \\
\hline \multicolumn{3}{|c|}{ Contribuciones no materiales } \\
\hline \multirow{6}{*}{$\begin{array}{l}\text { Aprendizaje } \\
\text { e inspiración }\end{array}$} & $\begin{array}{l}\text { Sistemas de captación de agua subálvea como } \\
\text { los qanats }\end{array}$ & Nasiri \& Mafakher (2015) \\
\hline & $\begin{array}{l}\text { Sistemas de captación de agua de la atmósfera } \\
\text { (por ejemplo, cosechas de niebla) }\end{array}$ & Alkhaddar (2003) \\
\hline & $\begin{array}{l}\text { Los paisajes áridos abiertos son especialmente } \\
\text { apropiados para la observación y el estudio } \\
\text { astronómico }\end{array}$ & Alberdi (2011) \\
\hline & $\begin{array}{l}\text { Los ríos secos contribuyen significativamente a } \\
\text { aumentar el conocimiento global de las ciencias } \\
\text { ambientales }\end{array}$ & Safriel \& Adeel (2015) \\
\hline & $\begin{array}{l}\text { Loa ríos secos son utilizados para desarrollar proyectos } \\
\text { de educación ambiental }\end{array}$ & $\begin{array}{l}\text { https://www.murcia.es/ } \\
\text { medio-ambiente/medio- } \\
\text { ambiente/publicaciones.asp }\end{array}$ \\
\hline & $\begin{array}{l}\text { Estos ecosistemas son inspiración para pintores, poetas } \\
\text { y escritores }\end{array}$ & Neruda (1976) \\
\hline \multirow{3}{*}{$\begin{array}{l}\text { Experiencias físicas } \\
\text { y psicológicas }\end{array}$} & $\begin{array}{l}\text { Los ríos secos permiten realizar actividades recreativas } \\
\text { como senderismo, caza, observación de especies } \\
\text { animales, etc. }\end{array}$ & Steward et al. (2012) \\
\hline & $\begin{array}{l}\text { Los ríos secos son atractivos para el turismo por su } \\
\text { biodiversidad y belleza escenográfica }\end{array}$ & Seely et al. (2003) \\
\hline & $\begin{array}{l}\text { Los paisajes áridos son espacios experienciales que } \\
\text { generan bienestar humano }\end{array}$ & $\begin{array}{l}\text { Andreu-Lara \& Ojeda- } \\
\text { Rivera (2019) }\end{array}$ \\
\hline
\end{tabular}




\begin{tabular}{|c|c|c|}
\hline $\begin{array}{l}\text { Contribuciones de los } \\
\text { ríos secos al bienestar } \\
\text { humano (NCP) }\end{array}$ & Ejemplos/evidencias & Referencias bibliográficas \\
\hline \multirow{6}{*}{ Soporte de identidad } & $\begin{array}{l}\text { Los ríos secos son utilizados como vías de } \\
\text { comunicación entre las poblaciones humanas, } \\
\text { confiriéndoles así una identidad que es reconocida } \\
\text { como propia por los habitantes locales, quienes llegan, } \\
\text { incluso, a asignarles nombres distintos }\end{array}$ & $\begin{array}{l}\text { Gómez et al. (2005); } \\
\text { Steward et al. (2012) }\end{array}$ \\
\hline & $\begin{array}{l}\text { La génesis de las tres principales religiones (judaísmo, } \\
\text { cristianismo e islam) está asociada con paisajes áridos }\end{array}$ & Burmil et al. (1999) \\
\hline & $\begin{array}{l}\text { Muchos elementos ambientales como las rocas y la } \\
\text { tierra en estos paisajes representan la piel de Dios para } \\
\text { los indios navajos }\end{array}$ & Kihonge (2017) \\
\hline & $\begin{array}{l}\text { Distintas especies de árboles, o algún árbol en } \\
\text { particular, tienen un fuerte significado espiritual para } \\
\text { las poblaciones locales }\end{array}$ & Safriel \& Adeel (2015) \\
\hline & $\begin{array}{l}\text { Las semillas del arbusto Anadenanthera colubrina } \\
\text { tienen un uso chamánico en las tierras áridas de } \\
\text { Sudamérica }\end{array}$ & Arenas (1992) \\
\hline & $\begin{array}{l}\text { Muchas formaciones geológicas tienen un fuerte } \\
\text { significado sagrado para las poblaciones humanas } \\
\text { locales }\end{array}$ & $\begin{array}{l}\text { Pungetti et al. (2012); } \\
\text { Samakov (2017) }\end{array}$ \\
\hline \multicolumn{3}{|c|}{ Mantenimiento de opciones } \\
\hline & $\begin{array}{l}\text { Las culturas nómadas se generaron en estos paisajes } \\
\text { y pueden ser una opción de futuro para una buena } \\
\text { calidad de vida }\end{array}$ & $\begin{array}{l}\text { Hillel (1991); Safriel \& } \\
\text { Adeel (2015); Root- } \\
\text { Bernstein et al. (2016) }\end{array}$ \\
\hline
\end{tabular}

De igual manera, las referencias relativas a las contribuciones materiales de los ríos secos revelan la diversidad de beneficios que los individuos y la sociedad obtienen para su bienestar. Estos beneficios son producidos por la naturaleza directamente sin intervención humana (por ejemplo, plantas medicinales; frutos silvestres) o coproducidos mediante la cooperación entre el sistema natural y social (por ejemplo, cultivos: Palomo et al., 2016) (tabla 1). Es obvio que la obtención directa de determinados recursos como, por ejemplo, alimentos para los humanos (Salinas y Guirado, 2002; Sánchez et al., 2004) o pastos para el ganado (Little et al., 1990; Arenas, 2012; Muiño, 2012; Martínez-Yoshino et al., 2021), energía (por ejemplo, leña o carbón: Ríos Saucedo et al., 2013; Martínez-Yoshino et al., 2021), materiales bióticos (por ejemplo, plantas medicinales: Ahmad et al., 2004; Arenas, 2012; Martínez-Yoshino et al., 2021; fibras y materiales para construcciones y artesanías: Linares et al., 2008) o materiales abióticos (por ejemplo, arena, oro...: Madyise, 2013; Crane, 2014; Albaladejo-García \& Gómez-Espín, 2016), en muchos casos tan solo cubre necesidades parciales o complementarias para el buen vivir, pero los modos de vida y las culturas de las poblaciones que viven en torno a estos ecosistemas han desarrollado, 
a lo largo de la historia, modelos de sostenibilidad en un proceso de coproducción acoplado a los ritmos naturales. Por ejemplo, los sistemas pastoriles tradicionales en las tierras áridas siempre fueron una solución para obtener alimentos de origen animal (Oteros-Rozas et al., 2013; Root-Bernstein et al., 2016) como alternativa más sostenible que la producción vegetal ligada a la tierra y a la disponibilidad de agua difícil de conseguir en ambientes áridos y semiáridos (Krätli et al., 2013). Estos sistemas llevan consigo modos de vida nómadas (por ejemplo la trashumancia estacional: Oteros-Rozas et al., 2014) acoplados a la variabilidad de las condiciones ambientales (Coughenour, 2004). Precisamente es la movilidad la que mantiene la resiliencia de estos ecosistemas. La búsqueda de alimento para el ganado en muchas ocasiones va ligada a utilizar vías que faciliten su traslado (como los cauces de los ríos secos: López Galán \& Muñoz, 2008) y donde aprovisionarse de agua (fuentes, pozos o charcas ganaderas acopladas a estos ríos y al subálveo: Vidal-Abarca et al., 2003; Martínez, 2004). El conocimiento ecológico local de los pastores y su capacidad de manejo de los escasos recursos en ambientes estresados es clave para que este sistema socioecológico conviva con las crisis ambientales y sea capaz de mantener un flujo de servicios ecosistémicos (Oteros-Rozas et al., 2012; Selemani, 2020).

Las contribuciones de regulación en el caso de los ríos secos se refieren a su capacidad para reducir la virulencia de las avenidas (Suftin et al., 2014), controlar parte de los ciclos biogequímicos (por ejemplo, secuestrando carbono inorgánico en los lechos secos: Farage et al., 2003), acelerar la descomposición del materia orgánica a través de procesos como la fotodegradación (Almagro et al., 2017; Del Campo \& Gómez, 2016), facilitar la infiltración del agua hacia los acuíferos (Suftin et al., 2014), regular el microclima local (Valenzuela et al., 2011; Roman, 2016), o la calidad del aire secuestrando nitrógeno total desde la atmósfera (Scholz et al., 2002), facilitar la formación de suelo (Ríos Saucedo et al., 2013) y de hábitats para muchas especies vegetales (Martínez-Yoshino et al., 2021) y animales (Williams et al., 2013; Sánchez-Montoya et al., 2016), favorecer la polinización (MartínezYoshino et al., 2021) y la dispersión de semillas (Reisener et al., 2018), e incluso controlar especies como, por ejemplo, hacen pequeños roedores sobre los árboles invasores del género Prosopis (Williams et al., 2013). Sin embargo, muchos de los ejemplos encontrados (tabla 1) se refieren bien a casos y/o experiencias locales, bien a evidencias aún no probadas. Las contribuciones de regulación están ligadas al funcionamiento de los ecosistemas y, en el caso de los ríos secos, aún se desconocen muchos de los procesos biogeoquímicos que los sustentan (Arce et al., 2019). Ni siquiera se tiene certeza de a qué escala espacial o temporal ocurren, o cuál es el papel funcional de las avenidas en la reactivación y/o desorganización de los cauces que son la base física que sustenta la provisión de estas contribuciones (Vidal-Abarca et al., 2020).

La clasificación de IPBES para los NCP incorpora una categoría especial denominada "mantenimiento de opciones" (tabla 1), que está vinculada a la vez con los tres grandes grupos de contribuciones: materiales, no materiales y regulación. Esta categoría hace referencia a aspectos relacionados con las oportunidades potenciales que ofrece la naturaleza, incluyendo el mantenimiento en el futuro de todas las NCP actuales y futuras que soporten la resiliencia de los ecosistemas y su capacidad para seguir proporcionando beneficios a la sociedad (Díaz et al., 2018). Los paisajes áridos, y en ellos los ríos secos, han generado a lo largo de la historia formas de vida y culturas acopladas a las posibilidades de usar los 
recursos naturales en común acuerdo con las pautas espaciales y temporales de la naturaleza manteniendo su resiliencia (Balbo et al., 2016; Andreu-Lara \& Ojeda-Rivera, 2019) (tabla 1). El agua es el elemento vital por excelencia. La obtención de agua para abastecimiento directamente de los ríos secos es difícil; sin embargo, las sociedades que viven en torno a estos han sabido aprovechar las distintas fases del ciclo del agua, obteniéndola directamente de la lluvia (Ballen Suárez et al., 2006; Torres, 2019), de la niebla (Alkhaddar, 2003), de las esporádicas avenidas que se suceden de forma impredecible (por ejemplo, boqueras: López Galán \& Muñoz, 2008; López Bermúdez, 2014) o de los acuíferos o subálveos que se acumulan bajo el lecho seco, mediante mecanismos a veces muy sofisticados (por ejemplo, los qanats: Nasiri \& Mafakheri, 2015; Gamero et al., 2017). Además, han sabido aprovechar el mayor grado de humedad y acumulación de materia orgánica en los lechos secos para cultivar cereales y obtener alimento para ellos o para su ganado (Hans et al., 1999; Rodríguez Vaquero, 2007) o practicar una "jardinería particular" para mantener poblaciones vegetales arbóreas que proveen muchos beneficios, como por ejemplo bosquetes de acacias (Acacia tortillis subsp. raddiana) que crecen en los cauces de los ríos secos (wadis) de Egipto y Sudán y que proporcionan madera, carbón vegetal, forraje para el ganado y sombra para todos (Andersen, 2007; Andersen et al., 2014).

La cantidad de estrategias y técnicas para ayudar a la regulación hídrica (es decir, controlar la ubicación, cantidad y tiempo en el que se puede acceder al agua tanto superficial como subterránea) es extraordinaria y muestra cómo en ecosistemas donde la falta de agua es lo habitual, la diversificación de los métodos de recogida y almacenaje del agua pueden sustentar modos de vida sostenibles (Bayly, 1999; Tahmasebi, 2009). Pero no solo la tecnología, sino también los modos de uso de los recursos (por ejemplo, el nomadismo: FAO, 2001) aprendidos a lo largo del tiempo (conocimiento ecológico local), los modelos de gobernanza para repartir solidariamente el agua y minimizar los conflictos (por ejemplo, el Alporchón en Vélez Blanco: Navarro Sánchez, 2010) e incluso los sistemas de creencias (Gozalo de Andrés, 2003) son básicos para mantener la resiliencia del sistema fluvial (Balbo et al., 2016). Pero además, este conocimiento ecológico local ha generado una alta diversidad de identidades culturales que se manifiestan en lenguas propias (según Safriel y Adeel, 2005, el 24 \% de los idiomas globales están asociados a ambientes áridos del planeta), fiestas y festejos ligados a los ríos secos (por ejemplo, romería de la Virgen de la Luz en la Rambla del Cañar: Sánchez Conesa, 2018; o la regata anual Henley-on-Todd, que tiene lugar en un río seco en el norte de Australia: Steward et al., 2012), creencias espirituales y religiosas (como las rogativas para pedir agua: Gozalo de Andrés, 2003) e inspiración artística (por ejemplo, la pintura de paisajes áridos: Andreu-Lara \& Ojeda-Rivera, 2019).

Finalmente, hay que señalar que existen conflictos o compromisos (trade-offs) entre muchas NCP de los ríos secos. Uno de los más habituales es el uso de los lechos de estos ecosistemas para la obtención de alimento (figura 1). Muchos cauces de los ríos secos acumulan mayor humedad y materia orgánica que sus alrededores, lo cual favorece el crecimiento de plantas anuales que, en muchos casos, sirven como pasto para el ganado y como tal se han utilizado a lo largo de la historia (Seely et al., 2003; Kihonge, 2017; Martinez-Yoshino et al., 2021). Es habitual que los lechos de los ríos secos sean utilizados para el cultivo de cereales o de algún tipo de arbolado de secano (como el algarrobo) (HernándezHernández \& Morales Gil, 2013), lo cual altera el sustrato, la vegetación que crece en estos e incluso el 
banco de semillas con el que restaurar la vegetación natural anual. El extremo de alteración se produce cuando sobre ellos se desarrolla una agricultura de regadío que no solo transforma y/o destruye el fondo de los lechos, sino que extrae el agua de los acuíferos para su mantenimiento comprometiendo muchas NCP (Skoulikidis et al., 2017).

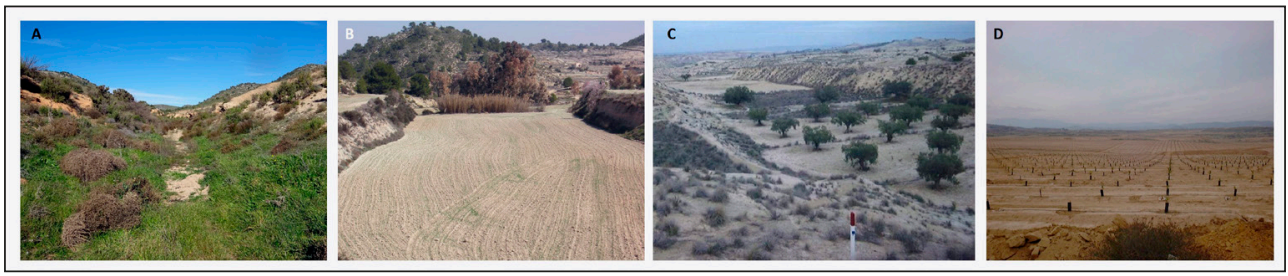

Figura 1. Ejemplo de usos del lecho de los ríos secos que generan compromisos.

A: lecho natural con crecimiento de plantas anuales usadas para la alimentación del ganado; B: lecho modificado y preparado para el cultivo de cereales; C: cultivo de arbolado de secano; D: desaparición del lecho del río seco para desarrollar una plantación de arbolado de regadío.

\section{Los principales impulsores de cambio}

De acuerdo con IPBES, los cinco impulsores de cambio directos sobre la biodiversidad y las NCP son la explotación de recursos naturales, el cambio de uso del suelo, la contaminación, el cambio climático y las especies exóticas invasoras (figura 2). Durante la revisión bibliográfica se han encontrado muchos ejemplos de todos ellos que alteran los ríos secos y su capacidad para contribuir al bienestar humano. El impulsor que mayor número de alteraciones produce sobre las NCP es el cambio de uso de suelo. Para el caso de los ríos secos, estos cambios implican la alteración parcial (por ejemplo, construcción de muros para evitar inundaciones o presas para acumular agua de escorrentía, o transformación del lecho para cultivar: Ito, 2005; Gómez et al., 2005) o total del propio cauce (por ejemplo, urbanizaciones sobre los ríos secos: Steward et al., 2012), incluida la modificación de su régimen de flujo natural (por ejemplo, por transferencias de agua desde otros cauces de flujo permanente: Briggs et al., 1993) que puede comprometer el abastecimiento de agua a las poblaciones locales. 


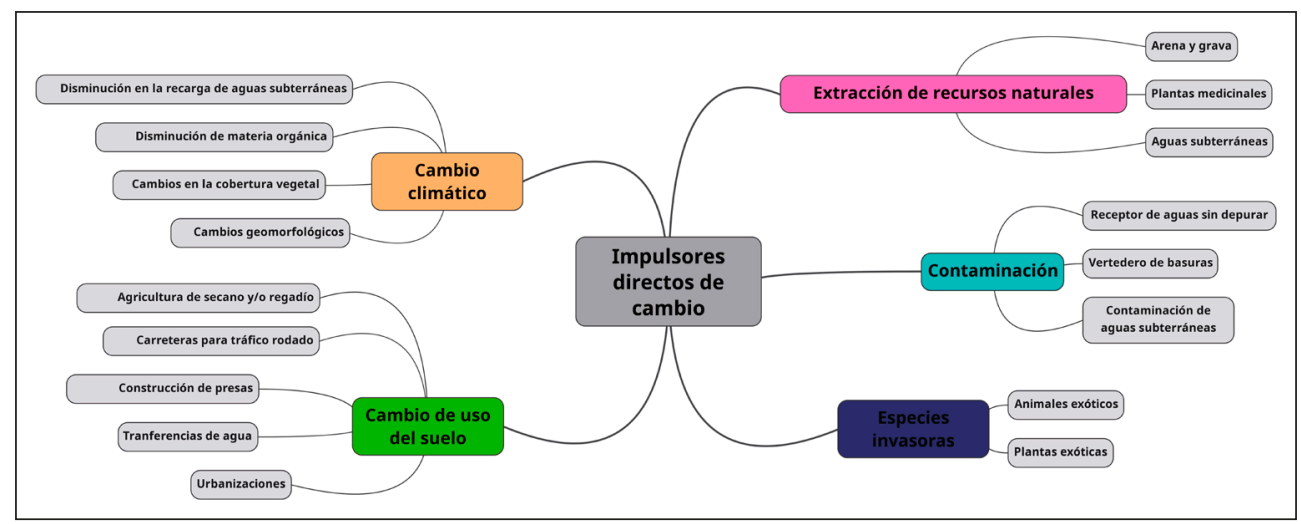

Figura 2. Principales impulsores directos de cambio detectados en la revisión bibliográfica que afectan a los beneficios de los ríos secos al bienestar humano.

La extracción de agua desde los acuíferos es un importante impulsor que modifica tanto la biodiversidad como la capacidad para mantener el flujo de muchos NCP a lo largo del tiempo (Pulford et al., 1992; Levick et al., 2008), lo que compromete el mantenimiento de la vegetación natural y, sobre todo, el acceso a fuentes de abastecimiento de agua (Levick et al., 2008). De igual manera, la extracción de arenas y gravas de los lechos de los ríos secos tiene importantes implicaciones en el control de la erosión, en la alteración del hábitat y en la disminución de su capacidad para potenciar la infiltración de agua a los acuíferos y minimizar las avenidas y la destrucción de comunidades vegetales utilizadas por las poblaciones locales como recursos alimenticios o como medicinas naturales (Madyise, 2013). Más recientemente se ha detectado la extracción masiva de plantas medicinales en cauces de ríos secos debido al boom actual de las poblaciones occidentales por el uso de la medicina tradicional, poniendo en peligro muchas plantas que viven en estos cauces y que de forma tradicional utilizan las comunidades locales (Ahmad et al., 2004).

El uso de los ríos secos como receptores de aguas residuales (Hassan \& Egozi, 2001) o como vertedero de basuras (Gómez et al., 2005) es un impulsor ampliamente extendido, uno de cuyos efectos más negativos es la contaminación de los acuíferos o subálveos que abastecen de agua tanto a poblaciones locales como a su ganado (Seely et al., 2003).

Es bien conocido que el impulsor directo de cambio climático acentuará las condiciones extremas (incremento de la temperatura y de la evapotranspiración, y disminución y comportamiento errático de las precipitaciones: Mirzabaev et al., 2019) que caracterizan a los ríos secos (por ejemplo, alargamiento de los periodos secos, menor humedad en los lechos y de disponibilidad de agua subterránea, etc.). La disminución de la vegetación es uno de los efectos más importantes sobre el bienestar humano porque es parte de la base de los sistemas agropastorales que se desarrollan en torno a estos cauces (como en el este de África: Mude et al., 2007; Pricope et al., 2013). Pero además, tanto la disminución de las precipitaciones como su desigual distribución afectan a la recarga de acuíferos (Squeo et al., 2006) que 
mantienen buena parte del servicio de abastecimiento de agua a las poblaciones locales. El cambio climático también afecta a muchas contribuciones de regulación, entre las que se señala la disminución de la materia orgánica que entra a los cauces (Almagro et al., 2010), el incremento de la salinidad en el lecho y de los procesos de erosión (Martínez-Mena et al., 2008) y cambios geomorfológicos que afectan al hábitat de las especies (Larkin et al., 2020).

Por último, las especies exóticas invasoras tanto vegetales como animales se ven favorecidas en los ríos secos por la alteración de las condiciones naturales que facilitan su asentamiento y expansión (Milton \& Dean, 2010; Williams et al., 2013; Zhang \& Jiang, 2016). La introducción de estas especies en muchos ecosistemas no siempre es accidental, sino que tiene una finalidad económica. Por ejemplo, Linders et al. (2020) estudian los efectos sobre varias NCP de la introducción de Prosopis en Kenia y Etiopía para aumentar la cantidad de forraje para el ganado y mejorar así la renta de las poblaciones locales. Estos autores demuestran que no solo no se ha conseguido el objetivo económico, sino que se han perdido muchas NCP, entre ellas la disminución de los pastos naturales, la reducción de la resistencia a la sequía, la disminución de la ganadería y del sistema cultural pastoril asociado a estos ecosistemas. Sin embargo, las especies invasoras pueden contribuir tanto de forma positiva como negativa (contraservicios: Shackleton \& Shackleton, 2018) al bienestar humano, lo cual depende de la percepción del usuario, como demuestran Tebboth et al. (2020) en un estudio sobre los impactos de Prosopis juliflora en Etiopía.

Pero a estos impulsores directos se superponen otros de carácter indirecto que, en muchas ocasiones, ponen en peligro la biodiversidad y la supervivencia de estos sistemas socioecológicos. IPBES reconoce cinco categorías de impulsores indirectos: económicos, institucionales, culturales y religiosos, demográficos y científicos/tecnológicos. Para el caso de los ríos secos, la dependencia de la gente de estos ecosistemas le ha llevado a establecer normas y prácticas tradicionales incorporando una ética del cuidado del entorno que ha generado costumbres y sabidurías específicas y diversidad de paisajes (Safriel \& Adeel, 2005; Sangha et al., 2018). Cuando la gestión local de un recurso escaso es embebido en un modelo económico concreto (por ejemplo, la globalización: Safriel \& Adeel, 2005) o por decisiones políticas diseñadas a gran escala, obviando la gobernanza consuetudinaria propia de estas comunidades humanas que allí habitan y de la que extraen sus recursos, se desvirtúa e ignora no solo el conocimiento ecológico local aprendido a lo largo de la historia, sino también su alta capacidad de autoorganización y cohesión interna de la comunidad, lo que lleva a la pérdida de la resiliencia del sistema y provoca una gran inequidad social (Merçon et al., 2019). En general, los sistemas socioecológicos generados en torno a los ríos secos están constituidos por poblaciones humanas de pequeño tamaño (Safriel \& Adeel, 2005; Balbo et al., 2016) con valores, creencias y normas sociales propias que suelen quedar al margen de la toma de decisiones políticas sobre la gestión de sus recursos. Por ejemplo, pocas veces los proyectos de conservación de espacios naturales han incluido la diversidad sociocultural de las poblaciones que allí habitan. En Australia, las exigencias de cincuenta pueblos indígenas han conseguido incorporar sus antiguas reglas al plan de manejo de sus territorios basadas en el mantenimiento de la relación gente-lugares-plantas y animales (Davies et al., 2013). 
El cambio global al que se encuentran sometidos todos los ecosistemas del planeta (Mauser et al., 2013) puede afectar con mayor intensidad a las poblaciones locales que viven en torno a los ríos secos (Balbo et al., 2016). Cabría pensar que la falta de agua por efecto del cambio climático será el factor más condicionante, pero probablemente son los impulsores socioeconómicos los que incidirán con mayor intensidad (Safriel \& Adeel, 2005). La capacidad para autoorganizarse es básica para mantener la sostenibilidad de estos ecosistemas (Balbo et al., 2016) y esto solo es posible si se respetan sus modelos de gobernanza tradicional. La integración de estas comunidades a la globalización expande los límites biofísicos en los que se sustentaba la sostenibilidad del sistema, lo cual lleva al desacoplamiento de estos sistemas socioecológicos (Balbo et al., 2016), una de cuyas consecuencias son las migraciones de parte de la población humana a zonas urbanas que la desvincula de sus conocimientos, raíces culturales y creencias. En este contexto, las mujeres son las grandes perdedoras (Ahmed et al., 2016; Chiblow, 2020).

Sin embargo, el gran elenco sociocultural que aportan estas comunidades a la gestión sostenible de los recursos que proporcionan los ríos secos puede ser especialmente interesante para abordar las crisis ambientales que afectan a buena parte del planeta. Una de las claves de la sostenibilidad de estos socioecosistemas está en el reconocimiento de las limitaciones biofísicas naturales que imperan en estos ecosistemas.

\section{Una aproximación a la percepción social y el conocimiento local de los ríos secos}

El método de libre listado dio como resultado una relación de 241 palabras y las seis más repetidas fueron: agua $(\mathrm{n}=18)$, seca $(\mathrm{n}=17)$, aburrida/o $(\mathrm{n}=12)$, interés/interesante $(\mathrm{n}=12)$, vegetación $(\mathrm{n}=12)$, peligro/peligrosa $(\mathrm{n}=10)$. Resulta curioso cómo los ríos secos son percibidos por la ciudadanía como un ecosistema seco, pero a la vez son relacionados con la presencia de agua y vegetación. Por otro lado, parece existir una polarización entre aquellas personas que lo consideran un ecosistema aburrido y quienes lo consideran interesante.

El análisis de la naturaleza de las palabras escogidas por la ciudadanía reveló tres enfoques de percepción distintos empleados en la descripción de las ramblas: empírico, utilitarista y sociocultural (figura 3). El enfoque empírico incluye aquellas palabras que hacen referencia a las estructuras, los factores o las funciones que caracterizan a los ríos secos (por ejemplo, natural, vegetación, erosión); el utilitarista agrupa aquellas palabras que se refieren a un beneficio o uso del ecosistema (como camino, aromáticas, vertidos), y el sociocultural, a las palabras que hacen referencia a factores sociales y culturales producto de la interacción entre el sistema natural y social y que está más ligado a las emociones y experiencia vitales que la gente tiene con estos ecosistemas (como peligroso, divertido, silencio). Del total de palabras utilizadas por los ciudadanos, 5 se consideraron ambiguas y se descartaron. De las 236 palabras restantes, 7 se ajustaron a dos enfoques diferentes (ver figura 4). Por ejemplo, la palabra cabras puede ser tanto un rasgo empírico como utilitarista; o agradables paseos puede expresar tanto un rasgo sociocultural como utilitarista. Por lo tanto, el total de palabras utilizadas fue de 243.

La mayoría de las palabras utilizadas por la ciudadanía para describir los ríos secos ( $\mathrm{n}=121 ; 50 \%)$ están relacionadas con las características empíricas de estos ecosistemas (figura 3A). En concreto, 
las palabras más utilizadas fueron agua $(n=18)$, secas $(n=17)$ y vegetación $(n=12)$ (figura 4). El agua, tanto su presencia como su ausencia y todos los fenómenos (por ejemplo, inundación, avenidas, aridez) o procesos relacionados con esta (por ejemplo, desbordamiento, turbio, sequedad, impredecible) fueron, con diferencia, las palabras más utilizadas. La vegetación fue el otro rasgo característico destacado por la ciudadanía (por ejemplo, matorral, poca flora, plantas, adelfa, cañas). Por el contrario, la fauna y la geomorfología fueron los elementos mencionados con menor frecuencia.

El enfoque utilitarista sobre la capacidad de estos ecosistemas para satisfacer las necesidades humanas fue el menos citado. De hecho, del total de palabras analizadas tan solo 30 (12\%) hacen referencia a su utilidad para el bienestar humano (figura 3A). La palabra más repetida fue útil $(n=7)$, seguida de camino $(n=3)$ y deforestado $(n=2)$ (figura 4$)$. Además, 12 de las palabras asignadas a este enfoque se refieren al uso de estos cauces como vertederos de residuos (como vertido, basura, escombros) o por su ocupación indebida (por ejemplo, invadida) (figura 3B), lo que evidencia el carácter marginal y peyorativo que la gente tiene de los ríos secos (DeLucio \& Múgica, 1994; García-Llorente et al., 2012). Estos usos generan impactos negativos en el ecosistema y afectan a otros usos tradicionales más sostenibles como la agricultura o el pastoreo (Seely et al., 2003; Gómez et al., 2005).

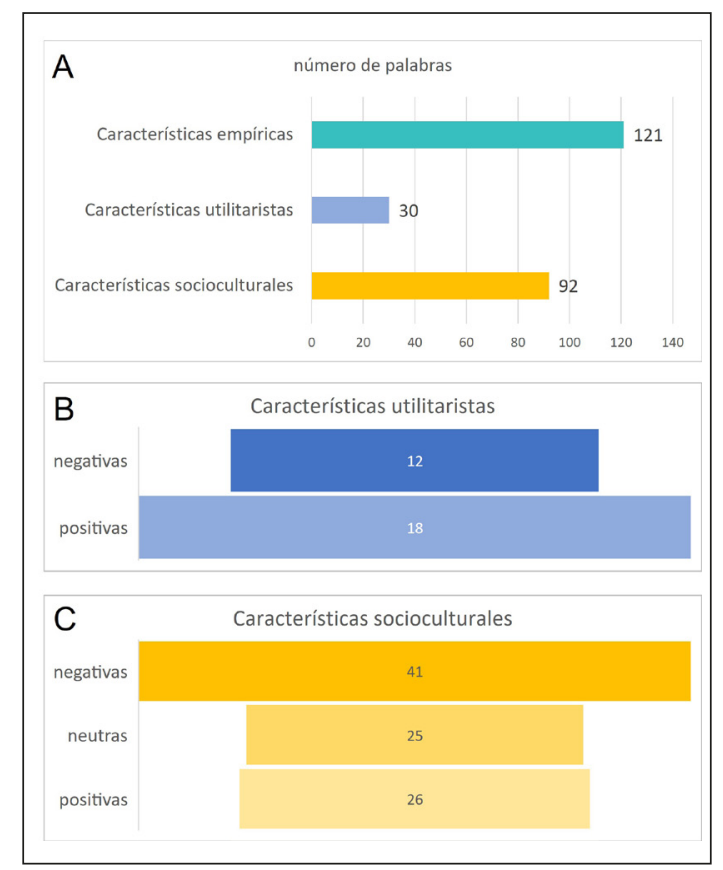

Figura 3. Número de palabras utilizadas por la ciudadanía para referirse a los ríos secos codificadas según las tres categorías descritas $(A)$. Desglose del número de las palabras asignadas a las categorías utilitaristas $(B)$ y socioculturales $(C)$. 


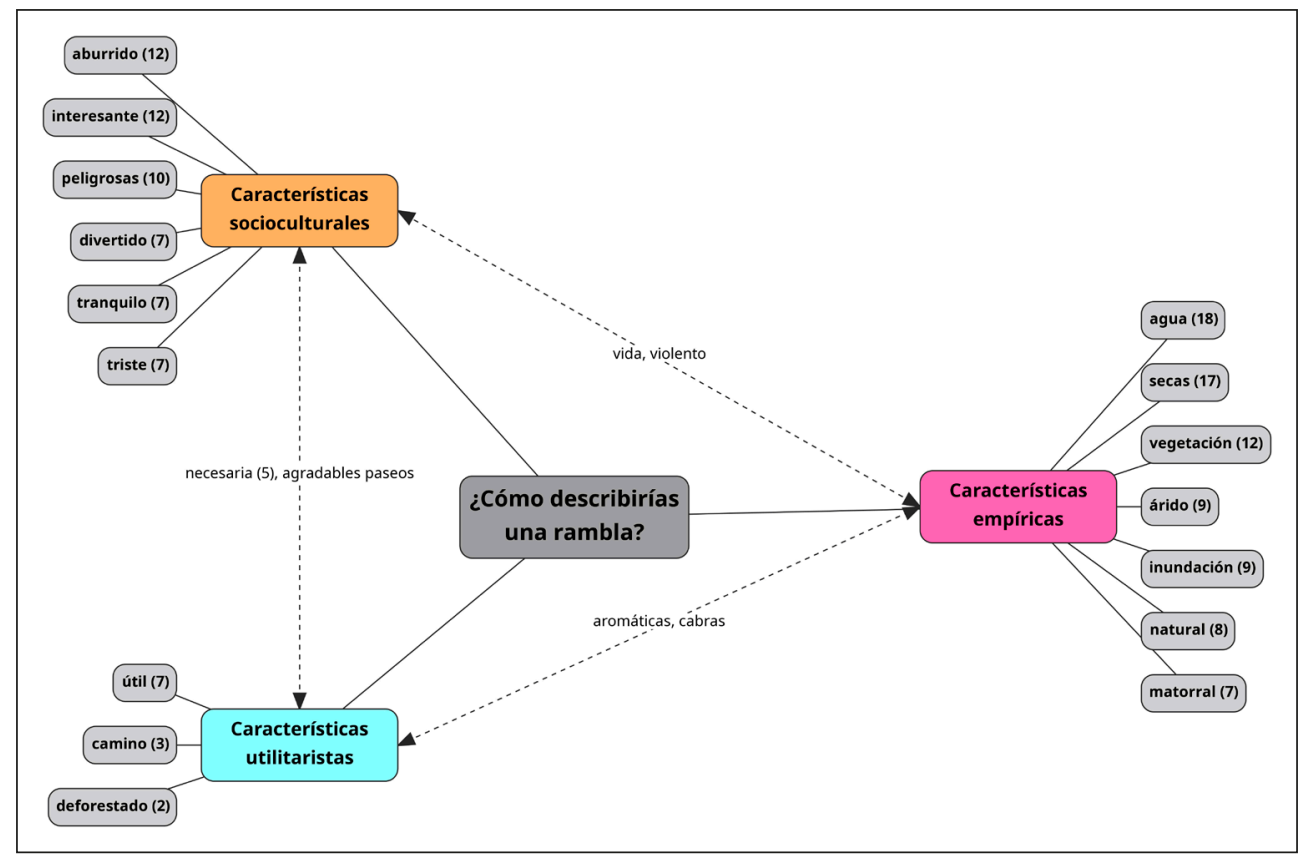

Figura 4. Principales palabras utilizadas por la ciudadanía para describir los ríos secos. Entre paréntesis se indica el número de veces que son usadas. Las dobles flechas indican palabras que pueden ser atribuidas a dos características diferentes.

Por último, hay que destacar el elevado número de palabras ( $\mathrm{n}=92 ; 38 \%$ del total) que pueden atribuirse a características socioculturales relacionadas con las vivencias de la ciudadanía (figura 3A). Las palabras más repetidas indican tanto experiencias positivas (divertido, tranquilo) como negativas (aburrida, peligrosa, triste), e incluso neutras (interesante, respeto) (figuras 3C y 4). Sin embargo, el mayor número de palabras $(\mathrm{n}=41)$ se relacionan con experiencias negativas (repulsión, terror, inhóspito, destrucción), muchas de las cuales llevan implícito las consecuencias no deseables que las avenidas de agua tienen sobre la población humana (Di Baldassarre et al., 2010; Machado et al., 2017).

\section{Conclusiones}

Los ríos secos son capaces de generar bienestar humano gracias a la diversidad de sus contribuciones materiales, no materiales y de regulación, al igual que ocurre con otros ecosistemas fluviales. Esta capacidad de los ríos secos para contribuir al bienestar humano está mantenida por el conocimiento ecológico de las poblaciones locales, que, a lo largo de la historia, han desarrollado modelos de "colaboración" entre el sistema natural y social. Sin embargo, esta coproducción de beneficios para el bienestar humano que se establece entre ambos sistemas es especialmente sensible a cambios políticos, 
económicos, sociales y ambientales, como el cambio climático. Por último, cabe señalar que parece existir un fuerte componente sociocultural o de conocimiento adquirido/aprendido que influye en las distintas formas en las que la ciudadanía percibe los ríos secos. En futuras investigaciones sería relevante estudiar el perfil de las personas que lo perciben de una u otra forma para averiguar si existen componentes de carácter social, demográfico o cultural detrás de estas percepciones. Es necesario profundizar en este aspecto, que puede ser de utilidad en los programas de conservación de estos singulares ecosistemas.

\section{Agradecimientos}

Este trabajo ha sido financiado por FEDER / Ministerio de Ciencia, Innovación y Universidades de España - Agencia Estatal de Investigación (AEI) / Proyecto CGL2017-84625-C2-2-R. Néstor Nicolás Ruiz disfruta de un contrato predoctoral de la Fundación Séneca (Ref: 20754/FPI/18).

\section{REFERENCIAS}

Ahmad, H., Bhatti, G. R., \& Latif, A. (2004). Medicinal flora of the Thar desert: an overview of problems and their feasible solutions. Zonas Áridas, 8, 73-83. http://dx.doi.org/10.21704/za,v8i1.715

Ahmed, A., Lawson, E. T., Mensah, A., Gordon, C., \& Padgham, J. (2016). Adaptation to climate change or non-climatic stressors in semi-arid regions? Evidence of gender differentiation in three agrarian districts of Ghana. Environmental Development, 20, 45-58. https://doi.org/10.1016/J. ENVDEV.2016.08.002

Albaladejo-García, J. A. \& Gómez-Espín, J. M. (2016). Espacios salineros de interior: Las salinas de Sangonera la Seca y Librilla en la Región de Murcia. Papeles de Geografía, 62, 34-46. http://dx.doi. org/10.6018/geografia/2016/241721

Alberdi, A. (2011). Grandes instalaciones en Astronomía: Astrofísica a lo grande. Lychnos, 5, 46-52.

Alkhaddar, R. M. (2003). Water harvesting in Jordan using earth ponds. Waterlines, 22(2),19-21. https://doi.org/10.3362/0262-8104.2003.05

Almagro, M., López, J., Boix-Fayos, C., Albaladejo, J., \& Martínez-Mena, M. (2010). Below-ground carbon allocation patterns in a dry Mediterranean ecosystem: A comparison of two models. Soil Biology and Biochemistry, 42, 1549-1557. https://doi.org/10.1016/j.soilbio.2010.05.031

Almagro, M., Martínez-López, J., Maestre, F. T., \& Rey, A. (2017). The contribution of photodegradation to litter decomposition in semiarid Mediterranean grasslands depends on its interaction with local humidity conditions, litter quality and position. Ecosystems, 20(3), 527-542. https://doi. org/10.1007/s10021-016-0036-5

Andersen, G. L. (2007). Long-term dynamics of wadi trees in a hyper-arid cultural Landscape. Tesis doctoral. University of Bergen. https://core.ac.uk/download/pdf/30925316.pdf. 
Andersen, G. L., Krzywinski, K., Talib, M., Saadallah, A. E.,M., Hobbs, J. J., \& Pierce, R. H. (2014). Traditional nomadic tending of trees in the Red Sea Hills. Journal of Arid Environments, 106, 36-44. http://dx.doi.org/10.1016/j.jaridenv.2014.02.009

Andreu-Lara, C. \& Ojeda-Rivera, J. F. (2019). La conciencia del paisaje. En Albelda, J. L., Sgaramella, C., \& Parreño, J. M. (eds.). Imaginar la transición hacia sociedades sostenibles (pp. 115-127). Valencia: Universitat Politécnica de Valencia.

Arce, M. I., Mendoza-Lera, C., Almagro, M., Catalan, N., Romani, A. M., Marti, E., Gómez, R., Bernal, S., Foulquier, A., Mutz, M., Marce, R., Zoppini, A., Gionchetta, G., Weigelhofer, G., del Campo, R., Robinson, C. T., Gilmer, A., Rulik, M., Obrador, B., Shumilova, O., Zlatanović, S., Arnon, S., Baldrian, P., Singer, G., Datry, T., Skoulikidis, N., Tietjen, B., \& von Schiller, D. (2019). A conceptual framework for understanding the biogeochemistry of dry riverbeds through the lens of soil science. Earth-Science Reviews, 188, 441-453. https://doi.org/10.1016/j.earscirev.2018.12.001

Arenas, P. (2012). Etnobotánica en zonas áridas y semiáridas del Cono Sur de Sudamérica. Consejo Nacional de Investigaciones Científicas-CONICET. Buenos Aires. http://www.ibiologia.unam.mx/ gela/etnobotanicaconosur.pdf.

Balbo, A. L., Gómez-Baggethun, E., Salpeteur, M., Puy, A., Biagetti, S., \& Scheffran, J. (2016). Resilience of small-scale societies: a view from drylands. Ecology and Society, 21(2). http://www. ecologyandsociety.org/volXX/issYY/artZZ/

Ballén Suárez, J. A., Galarza García, M. A., \& Ortiz Mosquera, R. O. (2006). Historia de los sistemas de aprovechamiento de agua de lluvia. VI SEREA - Semanario Iberoamericano sobre Sistemas de Abastecimento Urbano de Água João Pessoa (Brasil), 5 a 7 de junio de 2006. http://www.lenhs. ct.ufpb.br/html/downloads/serea/6serea/TRABALHOS/trabalhoH.pdf.

Bayly, I. A. E. (1999). Review of how indigenous people managed for water in desert regions of Australia. Journal of the Royal Society of Western Australia, 82,17-25.

Bieling, C., Plieninger, T., Pirker, H., \& Vogl, C.R. (2014). Linkages between landscapes and human well-being: An empirical exploration with short interviews. Ecological Economics, 105, 19-30. https://doi.org/10.1016/j.ecolecon.2014.05.013

Briggs, J., Dickinson, G., Murphy, K., Pulford, I., Esmat Belal, A., Moalla, S., Springuel, I., Ghabbour, S. I., \& Mekki, A. M. (1993). Sustainable development and resource management in marginal environments: natural resources and their use in the Wadi Allaqi region of Egypt. Applied Geography, 13, 259-284. https://doi.org/10.1016/0143-6228(93)90004-K

Bull, L. J. \& Kirkby, M. J. (2002). Dryland river characteristics and concept. En Bull, L. J. \& Kirkby, M. J. (eds.). Dryland Rivers: Hydrology and Geomorphology of the Semi-Arid Channels (pp. 3-15). Chichester, UK: John Wiley \& Sons.

Buttle, J. M., Boon, S., Peters, D. L., Spence, C., van Meerveld, H. J. (Ilja), \& Whitfield, P. H. (2012). An overview of temporary stream hydrology in Canada. Canadian Water Resources Journal, 37(4), 279-310. https://doi.org/10.4296/cwrj2011-903

Burmil, S., Daniel, T. C., \& Hetherington, J. D. (1999). Human values and perceptions of water in arid landscapes. Landscape and Urban Planning, 44, 99-109. 
Burns, R. G., DeForest, J. L., Marxsen, J., Sinsabaugh, R. L., Stromberger, M. E., Wallenstein, M. D., Weintraub, M. N., \& Zoppini, A. (2013). Soil enzymes in a changing environment: current knowledge and future directions. Soil Biology and Biochemistry, 58, 216-234. https://doi.org/10.1016/j. soilbio.2012.11.009

Chiblow, S. (2020). An indigenous research methodology that employs Anishiaabek elders, language speakers and women's knowledge for sustainable water governance. Waters, 12, 3058, https://doi. org/10.3390/w12113058

Crane, J. (2014). The environment in American history: Nature and the formation of the United States. Nueva York: Routledge. https://doi.org/10.4324/9781315817323

CONAZA-INE (Comisión Nacional de Zonas Áridas-Instituto Nacional de Ecología). (1994). Mezquite Prosopis spp. Cultivo alternativo para las zonas áridas y semiáridas de México. Comisión Nacional de las Zonas Áridas México, D.F. 31 p.

Coughenour, M. (2004). The Ellis paradigm - humans, herbivores and rangeland systems. African Journal of Range E Forage Science, 21(3), 191-200. https://doi.org/10.2989/10220110409485851

Datry, T., Larned, S. T., \& Tockner, K. (2014). Intermittent rivers: A challenge for freshwater ecology. Bioscience, 64, 229-235. https://doi.org/10.1093/biosci/bit027

Datry, T., Bonada, N., \& Boulton, A. (eds.) (2017a). Intermittent rivers and ephemeral streams. Ecology and management. Londres: Academic Press.

Datry, T., Boulton, A. J., Bonada, N., Fritz, K., Leigh, C., Sauquet, E., Tockner, K., Hugueny, B. \& Dahm, C. N. (2017b). Flow intermittence and ecosystem services in rivers of the Anthropocene. Journal of Applied Ecology, 55(1), 1-12. https://doi.org/10.1111/1365-2664.12941

Davies, J., Hill, R., Walsh, F. J., Sandford, M., Smyth, D., \& Holmes, M. C. (2013). Innovation in management plans for community conserved areas: experiences from Australian indigenous protected areas. Ecology and Society, 18(2), 14. http://dx.doi.org/10.5751/ES-05404-180214

DeLucio, J. V. \& Múgica, M. (1994). Landscape preferences and behaviour of visitors to Spanish national parks. Landscape Urban Planning, 29, 145-160.

Del Campo, R. \& Gómez, R. (2016). Exposure of wood in floodplains affects its chemical quality and its subsequent breakdown in streams. Science of the Total Environment, 543, 652-661. https://doi. org/10.1016/j.scitotenv.2015.11.050

Di Baldassarre, G., Montanari, A., Lins, H., Koutsoyiannis, D., Brandimarte, L., \& Blöschl, G. (2010). Flood fatalities in Africa: from diagnosis to mitigation. Geophysical Research Letters, 37(22), L22402. https://doi.org/10.1029/2010GL045467

Díaz, S., Demissew, S., Carabias, J., Joly, C., Lonsdale, M., Ash, N., Larigauderie, A., RamAdhikari, J., ... \& Zlatanova, D. (2015). The IPBES Conceptual Framework - connecting nature and people. Current Opinion in Environmental Sustainability, 14, 1-16. https://doi.org/10.1016/j. cosust.2014.11.002

Díaz, S., Pascual, U., Stenseke, M., Martín-López, B., Watson, R., Molnár, Z., Hill, R., Chan, K., Baste, I., Brauman, K., Polasky, S., Church, A., Lonsdale, M., Larigauderie, A., Leadley, P., Oudenhoven, A., Plaat, F., Schröter, M., Lavorel, S., Aumeeruddy-Thomas, Y., Bukvareva, E., Davies, K., 
Demissew, S., Erpul, G., Failler, P., Guerra, C., Hewitt, C., Keune, H., Lindley, S., \& Shirayama, Y. (2018). Assessing nature's contributions to people. Science, 359(6373), 270-272. https://dx.doi. org/10.1126/science.aap8826.

EME, Evaluación de los Ecosistemas del Milenio de España (2011). La Evaluación de los Ecosistemas del Milenio de España. Síntesis de resultados. Madrid: Fundación Biodiversidad. Ministerio de Medio Ambiente, y Medio Rural y Marino.

Farage, P., Pretty, J., \& Ball, A. (2003). Biophysical aspects of carbon sequestration in drylands. University of Essex Feb 03. http://www.fao.org/fileadmin/templates/nr/images/resources/pdf_documents/cseqbiophysicalaspectsdrylands.pdf

Food and Agriculture Organization of the United Nations (FAO) (2001). Pastoralism in the new millenium. Roma: FAO.

Fritz, K. M., Johnson, B. R., \& Walters, D. M. (2006). Field operations manual for assessing the hydrologic permanence and ecological condition of headwater streams, EPA/600/R-06/126. Washington, DC: U.S. Environmental Protection Agency, Office of Research and Development.

Gamero, F., Recio, J. M., García-Ferrer, A., \& Borja, C. (2017). Localización y caracterización de captaciones y antiguos Qanats de abastecimiento a la ciudad de Córdoba desde Sierra Morena. Boletín de la Asociación de Geógrafos Españoles, 74, 417-435. DOI: 10.21138/bage.2460.

García, C., Laronne, J. B., \& Sala, M. (1999). Variable source areas of bedload flux in a gravel-bed stream. Journal of Sediment Research, 69, 27-31.

García-Llorente M., Martín-López, B., Iniesta-Arandia, I., López-Santiago, C. A., Aguilera, P. A., $\&$ Montes, C. (2012). The role of multi-functionality in social preferences toward semi-arid rural landscapes: An ecosystem service approach. Environmental Science EO Policy, 19-20, 136-146. https://doi.org/10.1016/j.envsci.2012.01.006

Gómez, R., Hurtado, I., Suárez, M. L., \& Vidal-Abarca, M. R. (2005). Ramblas in south-east Spain: Threatened and valuable ecosystems. Aquatic Conservation, 15, 387-402. https://doi.org/10.1002/aqc.680

Gordon, N. D., McMahon, T. A., Finlayson, B. L., Gippel, C. J., \& Nathan, R. J. (2004). Stream Hydrology: An Introduction for Ecologists. Chichester, UK: Wiley.

Gozalo de Andrés, C. (2003). Meteorología popular. Las Rogativas. RAM, Revista del Aficionado a la Meteorologia, 15. https://www.divulgameteo.es/fotos/meteoroteca/Rogativas-Carmen-Gozalo.pdf.

Hans, R. K., Farooq, M., Babu, G. S., Srivastava, S. P., Joshi, P. C., \& Viswanathan, P. N. (1999). Agricultural produce in the dry bed of the River Ganga in Kanpur, India - a new source of pesticide contamination in human diets. Food and Chemical Toxicology, 37, 847-852. https://doi.org/10.1016/ s0278-6915(99)00066-6

Haines-Young, R. \& Potschin, M. (2013). Common international classification of ecosystem services (CICES). Consultation on Version 4, August-December 2012. Copenhague: European Environment Agency. https:// cices.eu/content/uploads/sites/8/2012/07/CICES-V43_Revised-Final_Report_29012013.pdf

Hassan, M. A. \& Egozi, R. (2001). Impact of wastewater discharge on the channel morphology of ephemeral streams. Earth Surface Processes and Landforms, 26, 1285-1302. https://doi.org/10.1002/ esp. 273 
Hernández-Hernández, M. \& Morales Gil, A. (2013). Los aprovechamientos tradicionales de las aguas de turbias en los piedemontes del sureste de la Península Ibérica: Estado actual en tierras alicantinas. Boletín de la Asociación de Geógrafos Españoles, 63, 105-123

Hillel, D. J. (1991). Out of the earth: civilization and the life of the soil. Nueva York: The Free Press.

Hinchman, G. (2013). Riparian vegetation and sandbar morphology along the lower Little Colorado River, Arizona. Physical Geography, 17(6), 534-553. https://doi.org/10.1080/02723646.1996.1064 2600

Ito, A. (2005). Distribution of the Nara plant that affect the life of the Topnaar people in the lower Kuiseb River, Namib Desert. African Study Monographs Supplementary issue, 30, 65-75.

Jacobson, P. J., Jacobson, K. M., \& Seely, M. K. (1995). Ephemeral Rivers and Their Catchments: Sustaining People and Development in Western Namibia. Windhoek, Namibia: Desert Research Foundation of Namibia.

Kadykalo, A. N., López-Rodriguez, M. D., Ainscough, J., Droste, N., Ryu, H., Ávila-Flores, G., Le Clec’h, S., Muñoz, M. C., Nilsson, L., Rana, S., Sarkar, P., Sevecke, K. J., \& Harmáčková, Z. V. (2019). Disentangling "ecosystem services" and "nature's contributions to people". Ecosystems and People, 15(1), 269-287. https://doi.org/10.1080/26395916.2019.1669713

Kelemen, E., García-Llorente, M., Pataki, G. Martín-López, B., \& Gómez-Baggethun, E. (2016). Nonmonetary techniques for the valuation of ecosystem services. En Potschin, M. \& Jax, K. (eds.). OpenNESS Reference Book EC FP7. Grant Agreement N. ${ }^{\circ}$ 308428. http://www.openness-project. eu/library/reference-book/sp-non-monetary-valuation

Kihonge, E. W. (2017). Assessing contribution of sacred natural sites to climate changes effects on dryland ecosystem case study: The Gabbra community, Marsabit County. Grado de Master en Ciencias. University of Nairobi.

Koundouri, P., Boulton, A. J., Datry, T., \& Souliotis, I. (2017). Ecosystem services, values, and societal perceptions of intermittent rivers and ephemeral streams. En Datry, T., Bonada, N., \& Boulton, A. (eds.). Intermittent Rivers and Ephemeral Streams, Ecology and Management (pp. 455-476). Londres: Academic Press.

Krätli, S., Huelsebusch, C., Brooks, S., \& Kaufmann, B. (2013). Pastoralism: A critical asset for food security under global climate change. Animal Frontiers, 3(1), 42-50. doi:10.2527/af.2013-0007

Larkin, Z. T., Ralph, T. J., Tooth, S., Fryirs, K. A., \& Carthey, A. J. R. (2020). Identifying threshold responses of Australian dryland rivers to future hydroclimatic change. Scientific Reports, 10, 6653. https://doi.org/10.1038/s41598-020-63622-3

Larned, S. T., Datry, T., Arscott, D. B., \& Tockner, K. (2010). Emerging concepts in temporary-river ecology. Freshwater Biology, 55, 717-738.

Levick, L. R., Goodrich, D. C., Hernandez, M., Fonseca, J., Semmens, D. J., Stromberg, J. C., Leidy, R. A., Scianni, M., Guertin, P. \& Kepner, W. G. (2008). The ecological and hydrological significance of ephemeral and intermittent streams in the arid and semi-arid American Southwest. Washington, DC: US Environmental Protection Agency, Office of Research and Development. 
Linares, E., Galeano, G., García, N., \& Figueroa, Y. (2008). Fibras vegetales utilizadas en artesanías en Colombia. Artesanías de Colombia S. A., Instituto de Ciencias Naturales. Facultad de Ciencias Universidad Nacional de Colombia. Sede Bogotá.

Linders, T. E. W., Bekele, K., Schaffner, U., Allan, E., Alamirew, T., Choge, S. K., Eckert, S., Haji, J., Muturi, G., Mbaabu, P. R., Shiferaw, H., \& Eschen, R. (2020). The impact of invasive species on social-ecological systems: Relating supply and use of selected provisioning ecosystem services. Ecosystem Services, 41, 101055. https://doi.org/10.1016/j.ecoser.2019.101055

Little, M. A., Dyson-Hudson, N., Dyson-Hudson, R., Ellis, J. E., Galvin, K. A., Leslie, P. W., \& Swift, D. M. (1990). Ecosystem approaches in human biology: Their history and a case of the south Turkana Ecosystem Project. En Moran, E.F . (ed.). The Ecosystem Approach in Anthropology: From Concept to Practice (pp. 389-434). Ann Arbor: The University of Michigan Press.

López Bermúdez, F. (2014). El riego por boquera en agricultura de secano, técnica hidráulica tradicional de lucha contra la desertificación en el sureste ibérico semiárido. En Geoecología, cambio ambiental y paisaje. Homenaje al profesor José María García Ruiz (pp. 405-414). Madrid: CSIC (Consejo Superior de Investigaciones Científicas).

López Galán, J. S. \& Muñoz, J. A. (coord.) (2008). Arquitectura tradicional. Guías de Almería. Territorio, Cultura y Arte. Instituto de Estudios Almerienses.

López-Rodríguez, M. D., Salinas-Bonillo, M. J., Torres, M. T., Pacheco-Romero, M., Guirado, E., Castro, H., \& Cabello, J. (2020). Impulsando estrategias colectivas ciencia-gestión-sociedad para conservar el hábitat de Ziziphus lotus (Hábitat Prioritario 5220). Ecosistemas, 29(1), 1890. https:// doi.org/10.7818/ECOS.1890

MA, Millennium Ecosystem Assessment (2005). Ecosystems and Human Well-being: Synthesis. Washington, DC: Island Press.

Mabbutt J. A. (1977). Desert Landforms. Cambridge, Mass.: MIT Press.

Machado, M. J., Medialdea, A., Calle, M., Rico, M. T., Sánchez-Moya, Y., Sopeña, A., \& Benito. (2017). Historical palaeohydrology and landscape resilience of a Mediterranean rambla (Castellon, NE Spain). Floods and people. Quaternary Science Reviews, 171, 182-198. ISSN 0277-3791. http:// hdl.handle.net/10261/190223

Madyise, T. (2013). Case studies of environmental impacts of sand mining and gravel extraction for urban development in Gaborone. Degree Master of Science. University of South Africa. https://core.ac.uk/ download/pdf/43174281.pdf

Martínez, M. L. (2004). Jurisdicción concejil y trashumancia en la baja Edad Media murciana. Murgetana, 110, 43-70.

Martínez-Mena, M., López, J., Almagro, M., Boix-Fayos, C., \& Albaladejo, J. (2008). Effect of water erosion and cultivation on the soil carbon stock in a semi-arid area of Southeast Spain. Soil $\mathbb{E}$ Tillage Research, 99, 119-129. https://doi.org/10.1016/j.still.2008.01.009

Martínez-Yoshino, N., Suárez, M. L., \& Vidal-Abarca, M. R. (2021). Delimitando los rasgos biológicos de la vegetación de los ríos secos: el caso de las ramblas de la cuenca del Segura (SE de España). Anales de Biología, 43, 11-25. http://dx.doi.org/10.6018/analesbio.43.02 
Mauser, W., Klepper, G., Rice, M., Schmalzbauer, B. S., Hackmann, H., Leemans, R., \& Moore, H. (2013). Transdisciplinary global change research: the co-creation of knowledge for sustainability. Current Opinion in Environmental Sustainability, 5, 420-431. http://dx.doi.org/10.1016/j.cosust.2013.07.001

Mengist, W., Soromessa, T., \& Legese, G. (2020). Ecosystem services research in mountainous regions: A systematic literature review on current knowledge and research gaps. Science of the Total Environment, 702, 134581. https://doi.org/10.1016/j.scitotenv.2019.134581

Merçon, J., Vetter, S., Tengö, M., Cocks, M., Balvanera, P., Rosell, J. A., \& Ayala-Orozco, B. (2019). From local landscapes to international policy: contributions of the biocultural paradigm to global sustainability. Global Sustainability, 2, e7, 1-11. https://doi.org/10.1017/sus.2019.4

Milton, S. J. \& Dean, W. R. J. (2010). Plant invasions in arid areas: special problems and solutions: a South African perspective. Biological Invasions, 12, 3935-3948. https://doi.org/10.1007/ s10530-010-9820-x

Mirzabaev, A., Wu, J., Evans, J., García-Oliva, F., Hussein, I. A. G., Iqbal, M. H., Kimutai, J., Knowles, T., Meza, F., Nedjraoui, D., Tena, F., Türkeş, M., Vázquez, R. J., \& Weltz, M. (2019). Desertification. En Shukla, P. R., Skea, J., Calvo Buendia, E., Masson-Delmotte, V., Pörtner, H.-O., Roberts, D. C., Zhai, P., Slade, R., Connors, S., van Diemen, R., Ferrat, M., Haughey, E., Luz, S., Neogi, S., Pathak, M., Petzold, J., Portugal Pereira, J., Vyas, P., Huntley, E., Kissick, K., Belkacemi, M., \& Malley, J. (eds.). Climate Change and Land: an IPCC special report on climate change, desertification, land degradation, sustainable land management, food security, and greenhouse gas fluxes in terrestrial ecosystems.

Mude, A., Ouma, R., Van de Steeg, J., Kaiuki, J., Opiyo, D., \& Tipilda, A. (2007). Kenya adaptation to climate change in the arid lands: Anticipating, adapting to and coping with climate risks in Kenya Operational recommendations for KACCAL. ILRI Research Report 18. Nairobi, Kenia: International Livestock Research Institute. https://hdl.handle.net/10568/2186

Muiño, W. P. (2012). Estudio etnobotánico de plantas usadas en la alimentación de los campesinos del noroeste de la Pampa Argentina. Chungara, Revista de Antropología Chilena, 44(3), 389-400. http:// dx.doi.org/10.4067/S0717-73562012000300003

Nasiri, F. \& Mafakheri, M. S. (2015). Qanat water supply systems: a revisit of sustainability perspectives. Environmental Systems Research, 4, 13. https://doi.org/10.1186/s40068-015-0039-9

Navarro Sánchez, Á. C. (2010). Una joya del derecho consuetudinario y de la hidráulica tradicional de la cuenca del Segura. Cangilón, 33, 340-382.

Neruda, P. (1976). Canto General. Biblioteca Ayacucho.

Oteros-Rozas, E., González, J. A., Martín-López, B., López, C. A. \& Montes, C. (2012). Ecosystem services and social-ecological resilience in transhumance cultural landscapes: learning from the past, looking for a future. En Plieninger, T. \& Bieling, C. (eds.). Resilience and the cultural landscape: understanding and managing change in human-shaped environments (pp. 242-260). Cambridge, UK: Cambridge University Press. http://dx.doi.org/10.1017/CBO9781139107778.018

Oteros-Rozas, E., Martín-López, B., López, C. A., Palomo, I., \& González, J. A. (2013). Envisioning the future of transhumant pastoralism through participatory scenario planning: a case study in Spain. The Rangeland Journal, 35(3), 251-272. https://doi.org/10.1071/RJ12092 
Oteros-Rozas, E., Martín-López, B., González, J. A., Plieninger, T., López, C. A., \& Montes, C. (2014). Socio-cultural valuation of ecosystem services in a transhumance social-ecological network. Regional Environmental Change, 14, 1269-1289. http://dx.doi.org/10.1007/s10113-013-0571-y

Palomo, I., Felipe-Lucia, M. R., Bennett, E. M., Martín-López, B., \& Pascual, U. (2016). Disentangling the pathways and effects of ecosystem service co-production. Advances in ecological research. En Woodward, G. \& Bohan, D. (eds.). Ecosystem services: from biodiversity to society, Part 2. Advances in ecological research (pp. 245-283). Amsterdam: Elsevier.

Parra, M. A., Enríquez, E., Ramírez, F., \& Martínez, M. (2013). Caracterización del Palo de Fierro (Olneya tesota) en Sonora, México. En Martínez, M. (ed.). Ecología y usos de especies forestales de interés comercial de las zonas áridas de México (pp. 193-217). Instituto Nacional de Investigaciones Forestales, Agrícolas y Pecuarias. Centro de Investigación Regional Norte-Centro. Sitio Experimental La Campana-Aldama. Aldama, Chih., México. http://biblioteca.inifap.gob. mx:8080/jspui/bitstream/handle/123456789/4067/CIRNOC_010106126600053621.pdf?sequence $=1$. (Consultado el 2-2-2021)

Pricope, N. G., Husak, G., Lopez-Carr, D., Funk, C., \& Michaelsen, J. (2013). The climate-population nexus in the East African Horn: emerging degradation trends in rangeland and pastoral livelihood zones. Global Environmental Change, 23, 1525-1541. https://doi.org/10.1016/J. GLOENVCHA.2013.10.002

Pulford, I. D., Murphy, K. J., Dickinson, G., Briggs, J. A., \& Springuel, I. (1992). Ecological resources for conservation and development in Wadi Allaqi, Egypt. Botanical Journal of the Linnean Society, 108, 131-141.

Pungetti, G., Oviedo, G., \& Hooke, D. (eds.) (2012). Sacred Species and Sites. Advances in Biocultural Conservation. Cambridge: Cambridge University Press. https://doi.org/10.1017/CBO9781139030717 Raymond, P., Hartmann, J., Lauerwald, R., Sobek, S., McDonald, C., Hoover, M., Butman, D., Striegl, R., Mayorga, E., Humborg, C., Kortelainen, P., Dürr, H., Meybeck, M., Ciais, P., \& Guth, P. (2013). Global carbon dioxide emissions from inland waters. Nature, 503, 355-359. https://doi. org/10.1038/nature12760

Región de Murcia Digital. Manuel Avellaneda. https://www.regmurcia.com/servlet/s. Sl?sit=c,371,m,1447\& r=ReP-1530-DETALLE_REPORTAJES (consultado el 28-1-2021).

Reiserer, R. S., Schuett, G. W., \& Greene, H. W. (2018). Seed ingestion and germination in rattlesnakes: overlooked agents of rescue and secondary dispersal. Proceedings of the Royal Society B: Biological Sciences, 285, 2017-2755. http://dx.doi.org/10.1098/rspb.2017.2755

Ríos Saucedo, J. C., Martínez Salvador, M., \& Mojica Guerrero, A. S. (2013). Caracterización ecológica y socioeconómica del mezquite (spp.). En Martínez, M. (ed.). Ecología y usos de especies forestales de interés comercial de las zonas áridas de México. Instituto Nacional de Investigaciones Forestales, Agrícolas y Pecuarias (pp. 42-68). Centro de Investigación Regional Norte-Centro. Sitio Experimental La Campana-Aldama. Aldama, Chihuahua, México. http://biblioteca.inifap.gob.mx:8080/jspui/bitstream/handle/123456789/4067/CIRNOC_010106126600053621.pdf?sequence=1 
Rodríguez Vaquero, J. E. (2007). Clasificación e ilustración de los paisajes hidráulicos de la cuenca del río Andaraz y los campos de Níjar (Almería). Nimbus, 19-20, 215-232

Roman, H. (2016). Bromatología de la vaina de mezquite (Prosopis spp.) como alternativa para consumo sustentable en la Comarca Lagunera. Universidad Autónoma Agraria Antonio Narro. http://repositorio.uaaan.mx:8080/xmlui/handle/123456789/7981.

Root-Bernstein, M., Guerrero-Gatica, M., Piña, L., Bonacic, C., Svenning, J.-C., \& Jaksic, F. M. (2016). Rewilding-inspired transhumance for the restoration of semiarid silvopastoral systems in Chile. Regional Environmental Change, 17, 1381-1396. https://doi.org/10.1007/s10113-016-0981-8

Safriel, U. \& Adeel, Z. (2005). Dryland systems, ecosystems and human well-Being: current state and rends. En Millenium Ecosystem Assessment. Current State Ë Trends Assessment (pp. 625-662). http:// www.millenniumassessment.org/documents/document.291.aspx.pdf

Salinas, M. J. \& Guirado, J. (2002). Riparian plant restoration in summer-dry riverbeds of Southeastern Spain. Restoration Ecology, 10, 695-702. https://doi.org/10.1046/j.1526-100X.2002.01050.x

Samakov, A. (2017). Spiritual commons: sacred sites as core of community-conserved areas in Kyrgyzstan. International Journal of the Commons, 11(1), 422-444. https://doi.org/10.18352/ijc.713

Sánchez, M. A., Sánchez-Zapata, J. A. \& Díez de Revenga, E. (2004). El conejo (Oryctolagus cuniculus) en la Región de Murcia. En ANSE (ed.). Actas del II Congreso de la Naturaleza de la Región de Murcia (pp. 169-179). Murcia: ANSE. http://www.naturalistas.es/masanchez/docs/El\%20Conejo\%20 en\%20la\%20R\%20de\%20Murcia.pdf

Sánchez Conesa, J. (2018). El Cañar, fiesta e identidad. https://www.laverdad.es/murcia/cartagena/canar-fiesta-identidad-20180105003246-ntvo.html?ref=https:\%2F\%2Fwww.google.com\%2F

Sánchez-Montoya, M. M., von Schiller, D., Ruhí, S. P. G., Proia, L., Miñano, J., Vidal-Abarca, M. R., Suárez, M. L., \& Tockner, K. (2016). Responses of ground-dwelling arthropods to surface flow drying in channels and adjacent habitats along Mediterranean streams. Ecohydrology, 9, 1376-1387. https://doi.org/10.1002/eco.1733

Sandercock, P., Hooke, J. M., \& Mant, J. M. (2007). Vegetation in dryland channels and its interaction with fluvial processes. Progress in Physical Geography, 31(2), 107-129. https://doi. org/10.1177/0309133307076106

Sangha, K. K., Preece, L., Villarreal-Rosas, J., Kegamba, J. J., Paudyal, K., Warmenhoven, T., \& RamaKrishnan, P. S. (2018). An ecosystem services framework to evaluate indigenous and local peoples' connections with nature. Ecosystem Services, 31, 111-125. https://doi.org/10.1016/j. ecoser.2018.03.017

Schneider, A., Jost, A., Coulon, C., Silvestre, M., Thery, S., \& Ducharne, A. C. G. (2017). Globalscale river network extraction based on high-resolution topography and constrained by lithology, climate, slope, and observed drainage density. Geophysical Research Letters, 44(6), 2773-2781. https://doi.org/10.1002/2016GL071844

Scholz, O., Gawne, B., Ebner, B., \& Ellis, I. (2002). The effects of drying and re-flooding on nutrient availability in ephemeral deflation basin lakes in Western New South Wales, Australia. River Research and Applications, 18, 185-196. https://doi.org/10.1002/rra.665 
Seely, M., Henderson, J., Heyns, P., Jacobson, P., Nakale, T., Nantanga, K., \& Schachtschneider, K. (2003). Ephemeral and endoreic river systems: relevance and management challenges. En Turton, A., Ashton, P., \& Cloete, E. (eds.). Transboundary rivers, sovereignty and development: hydropolitical drivers in the Okavango River Basin (pp. 187-212). Pretoria, South Africa: African Water Issues Research Unit.

Selemani, I. S. (2020). Indigenous knowledge and rangelands' biodiversity conservation in Tanzania: success and failure. Biodiversity and Conservation, 29, 3863-3876. https://doi.org/10.1007/ s10531-020-02060-z

Shackleton, S. E. \& Shackleton, R. T. (2018). Local knowledge regarding ecosystem services and disservices from invasive alien plants in the arid Kalahari, South Africa. Journal of Arid Environments, 159, 22-33. http://dx.doi.org/10.1016/j.jaridenv.2017.07.001

Skoulikidis, N. T., Sabater, S., Datry, T., Morais, M. M., Bu_agni, A., Dörflinger, G., Zogaris, S., Sánchez-Montoya, M. M., Bonada, N., Kalogianni, E., Rosado, J., Vardakas, L., De Girolamo, A. M., \& Tockner, K. (2017). Non-perennial Mediterranean rivers in Europe: status, pressures, and challenges for research and management. Science of the Total Environment, 577, 1-18. https://doi. org/10.1016/j.scitotenv.2016.10.147

Squeo, F. A., Aravena, R., Aguirre, E., Pollastri, A., Jorquera, C. B., \& Ehleringer, J. R. (2006). Groundwater dynamics in a coastal aquifer in north-central Chile: Implications for groundwater recharge in an arid ecosystem. Journal of Arid Environments, 67, 240-254. https://doi.org/10.1016/j. jaridenv.2006.02.01

Stanley, E. H., Fisher, S. G., \& Grimm, N. B. (1997). Ecosystem expansion and contraction in streams. Bioscience, 47(7), 427-435.

Steward, A. L., von Schiller, D., Tockner, K., Marshall, J. C., \& Bunn, S. E. (2012). When the river runs dry: human and ecological values of dry riverbeds. Frontiers in Ecology and the Environment, 10, 202-209. https://doi.org/10.1890/110136

Stubbington, R., Acreman, M., Acuña, V., Boon, P. J., Boulton, A. J., England, J., Gilvear, D., Sykes, T., \& Wood, P. J. (2020). Ecosystem services of temporary streams differ between wet and dry phases in regions with contrasting climates and economies. People and Nature, 2, 660-677. https:// doi.org/10.1002/pan3.10113

Sutfin, N. A., Shaw, J., Wohl. E. E., \& Cooper, D. (2014). A geomorphic classification of ephemeral channels in a mountainous, arid region, southwestern Arizona, USA. Geomorphology, 221, 164175. http://dx.doi.org/10.1016/j.geomorph.2014.06.005

Tahmasebi, A. (2009). Indigenous knowledge for water management in Iran's dry land - Siraf. International Journal of Environmental Studies, 66(3), 317-325. http://dx.doi. org/10.1080/00207230902722481

Tebboth, M. G. L., Few, R., Assen, M., \& Degefu, M. A. (2020). Valuing local perspectives on invasive species management: moving beyond the ecosystem service-disservice dichotomy. Ecosystem services, 42, 101068. https://doi.org/10.1016/j.ecoser.2020.101068

TEEB (2010). The Economics of Ecosystems and Biodiversity Ecological and Economic Foundations. Londres/Washington: Earthscan. 
Teff-Seker, Y. \& Orenstein, D. E. (2019). The "desert experience”: Evaluating the cultural ecosystem services of drylands through walking and focusing. People and Nature, 1, 234-248. https://doi. org/10.1002/pan3.28

Thompson, S. E., Assouline, S., Chen, L., Trahktenbrot, A., Svoray, T., \& Katul, G. G. (2014). Secondary dispersal driven by overland flow in drylands: review and mechanistic model development. Movement Ecology, 2, 7. http://www.movementecologyjournal.com/content/2/1/7

Torres, C. R. (2019). La captación del agua de lluvia como solución en el pasado y el presente. Ingeniería Hidráulica y Ambiental, XL(2), 125-139.

Valenzuela Núñez, L. M., Trucíos Caciano, R., Ríos Saucedo, J. C., Márquez Hernández, C., \& Rosales Serna, R. (2011). Dasometria de áreas mezquiteras en el norte-centro de México. En Ríos Saucedo, J. C., Trucíos Caciano, R., Valenzuela Núñez, L. M., Sosa Pérez, G., \& Rosales Serna, R. (eds.). Importancia de las poblaciones de mezquite en el norte-centro de México (pp. 135-172). INIFAP. CENID-RASPA.

Vidal-Abarca, M. R., Esteve, M. A., \& Suárez, M. L. (2003). Humedales y ramblas de la Región de Murcia. Comunidad Autónoma de Murcia y Fundación Universidad Empresa. http://www.murcianatural. carm.es/c/document_library/get_file?uuid=c0b4d1e8-ba98-4121-9d07-f5c3ccc9b9d3\& groupId=14

Vidal-Abarca, M. R., Gómez, R., Sánchez-Montoya, M. M., Arce, M. I., Nicolás, N., \& Suárez, M. L. (2020). Defining Dry Rivers as the Most Extreme Type of Non-Perennial Fluvial Ecosystems. Sustainability, 12, 7202. https://doi.org/10.3390/su1217720

Whitford, W. G. (2002). Ecology of desert systems. San Diego: Academic Press.

Williams, D., Pettorelli, N., Henschel, J., Cowlishaw, G., \& Douglas, C. M. S. (2013). Impact of alien trees on mammal distributions along an ephemeral river in the Namib Desert. African Journal of Ecology, 52. https://doi.org/10.1111/aje.12134

Zhang, L. \& Jiang, Z. (2016). Unveiling the status of alien animals in the arid zone of Asia. PeerJ, 4, e1545. https://doi.org/10.7717/peerj.1545

Cómo citar este artículo:

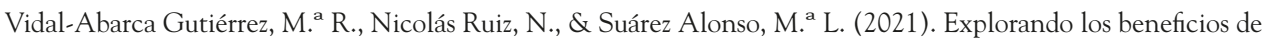
los ríos secos para el bienestar humano: Una perspectiva social. Cuadernos de Geografía, 107, 21-48.

https://doi.org/10.7203/CGUV.107.20783

\section{(c) $(1) \Theta \Theta$}

Este obra está bajo una licencia de Creative Commons Reconocimiento-NoComercial-SinObraDerivada 4.0 Internacional. 